\title{
The Effect of Physical Parameters on Flow Variables of an Electrically Conducting Viscoelastic Fluid
}

\author{
Binyam Zigta, Purnachandra Rao Koya* \\ School of Mathematical and Statistical Sciences, Hawassa University, Hawassa, Ethiopia
}

Email address:

tzigta@yahoo.com (B. Zigta),drkpraocecc@yahoo.co.in (P. R. Koya)

${ }^{*}$ Corresponding author

To cite this article:

Binyam Zigta, Purnachandra Rao Koya. The Effect of Physical Parameters on Flow Variables of an Electrically Conducting Viscoelastic Fluid. American Journal of Applied Mathematics. Vol. 5, No. 3, 2017, pp. 78-90. doi: 10.11648/j.ajam.20170503.13

Received: April 14, 2017; Accepted: April 27, 2017; Published: June 23, 2017

\begin{abstract}
In this paper the effect physical parameters on flow variables of unsteady, incompressible, electrically conducting viscoelastic fluid flowing between a pair of infinite vertical Couette porous channel walls embedded in a porous medium is analyzed. A uniform magnetic field is applied perpendicular to the channel walls. The temperature of the moving wall varies periodically. The temperature difference between the two walls is high enough due to thermal radiation. The solution of the governing equations is obtained using regular perturbation techniques. This technique is applied on partial differential equations that are difficult to solve. These partial differential equations are reduced to a set of ordinary differential equations in dimensionless form and thus they can be solved analytically. The effects of physical parameters on the flow variables are studied and the results have been discussed. The physical parameters considered include Hartmann number, viscoelastic parameter, Permeability of porous medium, chemical reaction parameter, radiative parameter, thermal Grashof number for heat transfer, modified Grashof number for mass transfer, frequency parameter, Prandtl number, mass diffusivity and Schmidt number. The flow variables considered include velocity, temperature and concentration. The theoretical results have been supported by simulation study. The observations include: (i) velocity decreases with increasing values of frequency, Hartmann number and viscoelastic parameter (ii) velocity increases with increasing values of temperature, thermal Grashof number, modified Grashof number and permeability of porous medium, (iii) the temperature decreases near the moving channel wall when the radiative parameter increases (iv) the temperature approaches to zero in the region near to the boundary layer of the stationary channel wall when the radiative parameter increases (v) concentration decreases with an increment in both chemical reaction and Schmidt number and (vi) The velocity of fluid increases as thermal Grashof number and modified Grashof number increases.
\end{abstract}

Keywords: Viscoelastic Fluid, MHD, Couette Channel Walls, Permeability of Porous Medium

\section{Introduction}

The change of shear stress versus strain rate inside a fluid depending on viscosity can be classified as a linear, nonlinear or plastic response. If the shear stress is linearly proportional to the strain rate then it is known as Newtonian fluid, and the constant of proportionality is called as the coefficient of dynamic viscosity. In case of non-Newtonian fluid, the shear stress is not only nonlinear to strain rate but also can be time dependent and hence coefficient of dynamic viscosity can't be a constant. The properties of non-Newtonian fluid flow are treated by rheology, the science of studying deformation and flow of a substance. A fluid is said to have Thixotropic property if its viscosity decreases only with time but neither with the shear stress nor with strain rate. Also, when shear stress is independent of strain rate then the fluid material possesses plastic deformation.

Viscoelastic is the property of a fluid material that combines both viscous and elastic characteristic while the fluid is undergoing deformation. The behavior of viscoelastic fluid is different from that of both Newtonian and inelastic non-Newtonian fluids. This behavior includes the presence of normal stresses in shear flows those are sensitive to deformation. Some materials that possess viscoelastic properties include: shampoos, hand creams, tooth pastes, 
yoghurts, polymers and paints. The factors that can affect the properties of viscoelastic are temperature, time and shear rate. Unsteady, incompressible, and electrically conducting 'viscoelastic fluid' embedded in a porous medium is of great importance. Such fluid has several applications in industry including polymer production, ceramics, lubrication, electromagnetic propulsion, and magneto-chemistry.

In literature it can be observed that there have been a very few studies conducted on the present subject. To fill the gap in this study it has been considered the effects of thermal radiation, chemical reaction, Permeability of porous medium and electrically conducting viscoelastic fluid. However, second order effects in elasticity, plasticity and fluid dynamics have been analyzed [1]. The Magneto hydrodynamic free convection laminar flow of incompressible viscoelastic fluid was studied [2]. The MHD combined free and forced convection flow through two parallel porous walls were studied [3]. An oscillatory mixed convection flow of electrically conducting viscoelastic fluid in an infinite vertical channel filled with porous medium was analyzed [4]. MHD free convection flow of viscoelastic fluid past an infinite porous plate was investigated [5]. The unsteady flow and heat transfer through an elastic viscous liquid along an infinite hot vertical porous moving plate with variable free stream and suction were examined [6]. Flow and heat transfer of an electrically conducting viscoelastic fluid between two horizontal squeezing and stretching plates have been studied [7]. Heat and mass transfer in MHD viscoelastic fluid flow through a porous medium over a stretching sheet with chemical reaction was studied [8]. MHD mixed convective viscoelastic fluid flow in a permeable vertical channel with Dufour effect and chemical reaction parameter was studied [9]. Viscoelastic fluid flow past an infinite vertical porous plate in the presence of first order chemical reaction was investigated [10]. MHD flow through a porous medium past a stretched vertical permeable surface in the presence of heat source or sink and a chemical reaction has been studied [11]. The three dimensional free convection flows through porous medium in a vertical channel with heat source and chemical reaction was studied [12]. The unsteady two dimensional flows and heat transfer through an elastic viscous liquid along an infinite hot vertical porous surface bounded by porous medium was studied [13]. MHD flows in non-Newtonian fluids has been studied [16].

The effects of radiation on free convection MHD flow have attracted many scholars due to its applications in space science. Such radiations occur due to high temperatures and design of relevant equipments. Furthermore, heat and mass transfer with thermal radiation on free convection MHD flow are significant in the plate heat transfer. Recently, many scholars focused on this field of study due to its applications in gas cooled nuclear reactors, nuclear power plants, gas turbines, space vehicles, and supersonic flights. The unsteady convective flow in a moving plate with thermal radiation was studied [17-18]. The combined effects of radiation and buoyancy force past a vertical plate has been studied [19]. The influence of thermal radiation on convective flows over a porous vertical plate was analyzed [20]. The effects of thermal radiation on heat and mass transfer over a moving vertical plate were studied [21]. The effects of thermal radiation on mixed convection flow over a permeable vertical plate with magnetic field have been studied [22].

Studies of energy and concentration transfer due to chemical reaction have significant applications in many branches of science and technology. The effects of concentration transfer with the involvement of chemical reaction are important in chemical process which helps to obtain high value products from cheaper raw materials. The quality of production and the flow behavior of the fluid is affected by the concentration goes through some kind of chemical reaction with the surrounding fluid.

The effects of mass transfer over a vertical oscillating plate with chemical reaction were studied [23]. Chemical reaction and thermal radiation effects on MHD convective flow in a porous medium in the presence of suction studied [24]. The effects of magnetic field on MHD Couette flow of a thirdgrade fluid with chemical reaction studied [25]. Even though, the effects of thermal radiation, chemical reaction, permeability of porous medium and electrically conducting viscoelastic fluid in vertical Couette channel wall embedded in a porous medium are important they have not been considered in these studies [15]. Hence the present study has been motivated to verify such effects.

The fluid considered here has the properties viz., unsteady, incompressible, electrically conducting viscoelastic, chemical reacting and thermal radiating. Free stream velocity of the fluid is assumed to be fluctuating. Furthermore, it is assumed that temperature and concentration of the fluid do also fluctuate with time. The main objective of the present study is to analyze the effect of physical parameters on flow variables of unsteady, incompressible, and electrically conducting viscoelastic fluid on free convection oscillatory fluid flow bounded between two infinite vertical Couette channel walls embedded in a porous medium when the temperature and concentration oscillates with time in the presence of thermal radiation, chemical reaction and permeability of porous medium.

\section{Mathematical Modeling and Analysis of the Problem}

Note that here and in what follows fluid means unsteady, incompressible, electrically conducting viscoelastic fluid, chemical reacting and radiating fluid.

Consider two dimensional free convection Couette fluid flows bounded by two infinite vertical channel walls separated by a distance $b$ embedded in a porous medium. The geometrical representation of the model and coordinate system are shown in Figure 1. The $\bar{x}$ axis is parallel to the gravitational acceleration vector $g$ but in opposite direction. The $\bar{y}$ axis is transverse to the channel walls.

The vertical moving channel wall is located at $\bar{y}=0$ along $\bar{x}$ where the temperature is $\bar{T}_{m}$ and the concentration is $\bar{C}_{m}$. The other stationary channel wall is located at $\bar{y}=b$ where 
the temperature is $\bar{T}_{b}$ and the concentration is $\bar{C}_{b}$.

Initially at $\bar{t}=0$, the stationary channel wall and the fluid are at the same temperature $\bar{T}_{b}$ and concentration level of the fluid $\bar{C}_{b}$ is the same at all points. At $\bar{t}>0$, the temperature of the moving wall and concentration of the fluid are raised to $\bar{T}_{m}$ and $\bar{C}_{m}$ respectively and they are maintained at constant values. This result is due to porous medium i.e., porous medium is used to insulate a heated body by maintaining its temperature.

As time changes temperature changes leading to buoyancy force acting in fluid flow so the channel wall at $\bar{y}=0$ starts moving in its own plane along positive $\bar{x}$ direction with velocity $V$. And for all the later times $\bar{t}>0$ the channel wall continues to be in motion. But due to viscosity of the fluid and acceleration due to gravity the velocity will decrease and consequently it starts moving in opposite direction after some time $\bar{t}$ the moving channel wall being heated by supplying external heat so as to maintain its temperature constant $\bar{T}_{m}$. However, the temperature of the wall at $\bar{y}=b$ rises to an upper constant value $\bar{T}_{b}$.

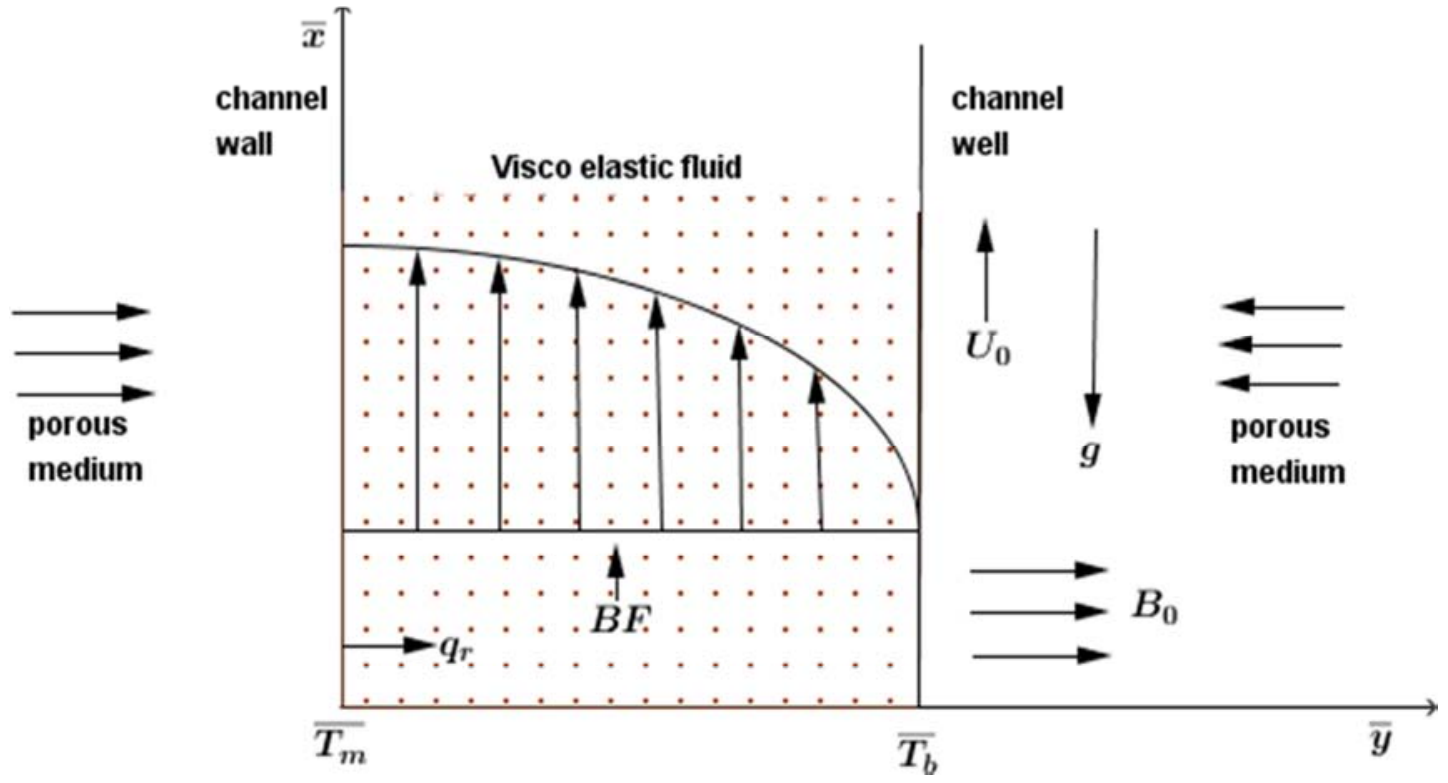

Figure 1. Geometrical representation of the model.

The governing equations of the model are derived based on the following assumptions: (i) The fluid properties such as temperature, viscosity, pressure, specific volume, specific gravity are constant (ii) the density varies with both temperature and concentration in the body force term or Boussinesq's approximation. It is a variable but not a constant (iii) the flow of the fluid is unsteady, laminar, incompressible, electrically conducting viscoelastic, thermal radiating and chemical reacting (iv) the magnetic field applied is transversal between the channel walls (v) the induced magnetic field of the fluid is negligible and hence the magnetic Reynolds number is a very small constant, the external electric field is supposed to be zero (vi) the electric field due to polarization of charges is negligible (vii) viscosity is considered with the constant permeability of porous medium (viii) there exists a homogeneous chemical reaction of first order with constant rate between diffusing concentration or species and the fluid in the moving wall. Note that a reaction is said to be of first order if the rate of reaction is directly proportional to concentration.

The expression for free stream velocity can be formed as

$$
\bar{U}(\bar{t})=U_{o}\left(1+\varepsilon e^{i \bar{\omega} \bar{t}}\right)
$$

Here in (1), $U_{o}$ is the mean constant free stream velocity, $\bar{\omega}$ is the frequency and $\bar{t}$ is the time. We now derive the governing equations of the present model. The momentum equation of the model takes the form as

$$
\frac{\partial \bar{u}}{\partial \bar{t}}=\frac{\partial \bar{U}}{\partial \bar{t}}+v \frac{\partial^{2} \bar{u}}{\partial \bar{y}^{2}}+g \beta\left(\bar{T}-\bar{T}_{b}\right)+g \beta_{c}\left(\bar{C}-\bar{C}_{b}\right)-\frac{J X B}{\rho}-\frac{v \bar{u}}{\bar{k}}+\frac{\bar{k}_{o}}{\rho} \frac{\partial \tau_{x y}}{\partial \bar{y}}
$$

In (2), the vector cross product $J x B$ is the Lorentz force. This term is a body force corresponding to magneto hydrodynamics flow. The minus sign in $-(v \bar{u} / \bar{k})$ indicates that the fluid flows along the direction from higher to lower potential. The plus sign in shear stress of viscoelastic fluid $\left[\left(\bar{k}_{0} / \rho\right)\left(\partial \tau_{x y} / \partial \bar{y}\right)\right]$ is tensile stress or pulling force. Using ohm's law, the expression for the density of current can be constructed $J=\sigma(E+\bar{v} \times B)$. Upon substituting $E=0$, since electric field is assumed to be negligible, it reduces to $J=\sigma(\bar{v} \times B)$. Also, the expression for Lorentz force reduces and takes the form as $J x B=-\sigma B^{2} \bar{u}$. In view of these result, the equation (2) reduces to

$$
\frac{\partial \bar{u}}{\partial \bar{t}}=\frac{\partial \bar{U}}{\partial \bar{t}}+v \frac{\partial^{2} \bar{u}}{\partial \bar{y}^{2}}+g \beta\left(\bar{T}-\bar{T}_{b}\right)+g \beta_{c}\left(\bar{C}-\bar{C}_{b}\right)-\frac{\sigma B^{2}(\bar{u}-\bar{U})}{\rho}-\frac{v \bar{u}}{\bar{k}}+\frac{\bar{k}_{o}}{\rho} \frac{\partial \tau_{x y}}{\partial \bar{y}}
$$


In (3) $\tau_{x y}$ is the component of the shear stress of the viscoelastic fluid given in [26] as

$$
\tau_{x y}=\left[\mu(\partial \bar{u} / \partial \bar{y})-(\mu / \eta)\left(\partial \tau_{x y} / \partial \bar{t}\right)\right] .
$$

Here $\mu$ is the coefficient of dynamic viscosity and $\eta$ is the modulus of rigidity. If $\eta$ approaches to infinity or if the component of shear stress is independent of time that is at steady state, the fluid behaves like a viscous fluid without elasticity. Solving for $\tau_{x y}$ in terms of the velocity component $\bar{u}$ we obtain

$\frac{\partial \tau_{x y}}{\partial \bar{y}}=\frac{\partial}{\partial \bar{y}}\left(\mu \frac{\partial \bar{u}}{\partial \bar{y}}\right)-\frac{1}{\alpha} \frac{\partial}{\partial \bar{y}}\left[\mu \frac{\partial}{\partial \bar{t}}\left(\mu \frac{\partial \bar{u}}{\partial \bar{y}}\right)\right] \approx \mu \frac{\partial^{2} \bar{u}}{\partial \bar{y}^{2}}-\frac{\mu^{2}}{\alpha}\left(\frac{\partial^{3} \bar{u}}{\partial \bar{t} \partial \bar{y}^{2}}\right)$

The higher order terms are neglected since their contributions are small enough as $\eta$ approaches to infinity. In view of these the equation (3) after some algebraic manipulations reduces to

$$
\begin{gathered}
\omega\left(\frac{\partial u}{\partial t}\right)=\omega\left(\frac{\partial U}{\partial t}\right)+\left[1+\frac{k_{0} v \mu}{U_{0}{ }^{2}}\right] \frac{\partial^{2} u}{\partial y^{2}}+G r \theta+G c C-M^{2}(u-U) \\
-\frac{U_{0}^{2} u b^{2}}{k v^{2}}-\frac{k_{0} v^{2} \mu^{2} \omega}{U_{0}^{2} \alpha b^{2}} \frac{\partial^{3} u}{\partial t \partial y^{2}}
\end{gathered}
$$

The energy equation of the model can be expressed as

$$
\frac{\partial \bar{T}}{\partial \bar{t}}=\alpha \frac{\partial^{2} \bar{T}}{\partial \bar{y}^{2}}-\frac{1}{\rho C_{p}} \frac{\partial q_{r}}{\partial y}
$$

The concentration equation of the model can be expressed as

$$
\frac{\partial \bar{C}}{\partial \bar{t}}=D \frac{\partial^{2} \bar{C}}{\partial \bar{y}^{2}}-k_{r}\left(\bar{C}-\bar{C}_{b}\right)
$$

The variables and parameters used in this study and their meanings are as follows: $G r$ thermal Grashof number; Gc modified Grashof number; $S c$ Schmidt number; $t$ time; $M$ Hartmann number; Pr Prandtl number; $q_{r}$ radiative heat flux; $k_{0}$ viscoelastic parameter; $k_{r}$ chemical reaction parameter; $R$ radiation parameter; $D$ mass diffusivity; $\bar{T}$ temperature of the fluid in the boundary layer; $\bar{T}_{m}$ temperature of the moving channel wall; $\bar{T}_{b}$ temperature of the stationary channel wall; $\bar{U}$ free stream velocity; $U$ dimension less free stream velocity; $\bar{u}$ velocity component in $\bar{x}$ direction; $\bar{v}$ velocity component in $\bar{y}$ direction; $\bar{x}, \bar{y}$ Cartesian coordinates; $x, y$ dimension less Cartesian coordinates; $\bar{C}_{m}$ concentration at channel wall at $y=0 ; \bar{C}_{b}$ concentration at channel wall at $y=$ $b ; C$ dimension less concentration; $C_{p}$ Specific heat at constant pressure; $J$ Electric current density; $g$ acceleration due to gravity; $\beta$ thermal expansion coefficient; $\beta_{c}$ concentration expansion coefficient; $\varepsilon$ amplitude of free stream velocity; $\omega$ Frequency of oscillation; $\theta$ dimension less temperature; $\kappa$ Thermal conductivity; $v$ Kinematic viscosity; $\mu$ Dynamic viscosity; $\alpha$ Thermal diffusivity; and $\sigma$ Electric conductivity.
The set of equations (3) - (5) govern the present model represented in Figure 1.

In (4), the term $\left[\left(1 / \rho C_{p}\right)\left(\partial q_{r} / \partial y\right)\right]$ is the thermal radiation effect. The minus sign of the radiative heat flux term indicates the emission of energy away from the objects that is heat is lost. Furthermore, in this equation, $\alpha>0$ shows heat generation and $\alpha<0$ indicates heat absorption i.e., thermal energy diffuses more rapidly through substances with high thermal diffusivity $\alpha$ and it diffuses slowly through those with low thermal diffusivity $\alpha$.

In (5), the minus sign indicates that the mass diffusion represents 'Generative chemical reaction'. Here chemical reaction is an exothermic reaction and in that case heat is generated. However, the chemical reaction is said to be generative or exothermic if the chemical reaction parameter is negative i.e. $k_{r}<0$.If $k_{r}>0$ the chemical reaction is said to be destructive or endothermic chemical reaction. Also $k_{r}\left(\bar{C}-\bar{C}_{b}\right)$ is the generative chemical reaction term.

Based on Figure 1 the boundary conditions of the model (3) - (5) can be expressed as

$$
\begin{gathered}
\bar{y}=0, \bar{u}=U_{0}\left(1+\varepsilon e^{i \bar{\omega} \bar{t}}\right) \\
\bar{T}=\bar{T}_{m}+\varepsilon\left(\bar{T}_{m}-\bar{T}_{b}\right) e^{i \bar{\omega} \bar{t}} \\
\bar{C}=\bar{C}_{m}+\varepsilon\left(\bar{C}_{m}-\bar{C}_{b}\right) e^{i \bar{\omega} \bar{t}} \\
\bar{y}=b, \bar{u}=0, \bar{T}=\bar{T}_{b}, \bar{C}=\bar{C}_{b}
\end{gathered}
$$

It is assumed that the radiation heat flux is unidirectional along positive $\bar{y}$ direction. Using the Roseland approximation for radiative heat transfer and the Roseland approximation for diffusion and also following the other scholarly works [27], the expression for radiative heat flux can be given as $q_{r}=-\left(4 \sigma / 3 k_{s}\right)\left(\partial \bar{T}^{4} / \partial \bar{y}\right)$. Here, the parameters $\sigma$ and $k_{s}$ represent the Stefan Boltzmann constant and the Roseland mean absorption coefficient respectively.

We now assume that the temperature differences within the fluid flow are sufficiently small so that $\bar{T}^{4}$ can be expressed as a linear function of $\bar{T}_{b}$ using Taylor series expansion. The Taylor series expansion of $\bar{T}^{4}$ about $\bar{T}_{b}$, after neglecting the higher order terms, takes the form as $\bar{T}^{4} \cong 4 \bar{T}_{b}{ }^{3} \bar{T}-3 \bar{T}_{b}{ }^{4}$. Using $q_{r}$ and approximation of $\bar{T}^{4}$ in (4), we obtain

$$
\frac{\partial \bar{T}}{\partial \bar{t}}=\alpha\left(\frac{\partial^{2} \bar{T}}{\partial \bar{y}^{2}}\right)+\left(\frac{1}{\rho C_{p}}\right)\left(\frac{16 \sigma \bar{T}_{b}{ }^{3}}{3 k_{s}}\right)\left(\frac{\partial^{2} \bar{T}}{\partial \bar{y}^{2}}\right)+\left(\frac{Q_{0}}{\rho C_{p}}\right)\left(\bar{T}-\bar{T}_{b}\right)
$$

Here in (10), the term $\left[\left(Q_{0} / \rho C_{p}\right)\left(\bar{T}-\bar{T}_{b}\right)\right]$ denotes heat absorption of the fluid.

\section{Non Dimensionalization of the Model}

In order to solve the governing equations (3) - (5) of the model it is convenient and easy to deal with its dimensionless form. Hence, we find the dimensionless form of the model by introducing the following non dimensional quantities. 


$$
\begin{gathered}
y=\bar{y} / b, R=4 \sigma \bar{T}_{b}{ }^{3} / \kappa k_{s}, u=\bar{u} / U_{0}, U=\bar{U} / U_{0}, t=\bar{\omega} \bar{t}, \varpi=\bar{\omega} b^{2} / v, \operatorname{Pr}=v / \alpha, S c=v / D, \\
G c=\left\{\left[g \beta_{c} b^{2}\left(\bar{C}_{m}-\bar{C}_{b}\right)\right] / v U_{0}\right\}, G r=\left[g \beta b^{2}\left(\bar{T}_{m}-\bar{T}_{b}\right) / v U_{0}\right], M=\sqrt{\left(\sigma B^{2} b^{2} / \rho v\right)} \\
, C=\left[\left(\bar{C}-\bar{C}_{b}\right) /\left(\bar{C}_{m}-\bar{C}_{b}\right)\right], k=\bar{k} U_{0}{ }^{2} / v^{2}, k_{o}=\frac{\bar{k}_{o} U_{0}{ }^{2}}{\rho v^{2}}, \theta=\left[\left(\bar{T}-\bar{T}_{b}\right) /\left(\bar{T}_{m}-\bar{T}_{b}\right)\right]
\end{gathered}
$$

After substituting the above non-dimensional quantities in (3) - (5) and after simple algebraic manipulations, the nondimensional form of the model takes the following form as

$$
\begin{aligned}
& \omega \frac{\partial u}{\partial t}=\omega \frac{\partial U}{\partial t}+\left(1+\frac{k_{0} v \mu}{U_{0}{ }^{2}}\right) \frac{\partial^{2} u}{\partial y^{2}}+G r \theta+G c C-M^{2}(u-U)-\frac{U_{0}^{2} u b^{2}}{k v^{2}}-\frac{k_{0} v^{2} \mu^{2} \omega}{U_{0}^{2} \alpha b^{2}} \frac{\partial^{3} u}{\partial t \partial y^{2}} \\
& \omega \frac{\partial \theta}{\partial t}=\frac{1}{p r}\left(1+\frac{4 R}{3}\right) \frac{\partial^{2} \theta}{\partial y^{2}}+\frac{Q_{o}}{\rho C_{p} v} \theta h^{2} \\
& S c \frac{\partial C}{\partial t}=\frac{\partial^{2} C}{\partial y^{2}}-\frac{K_{r} C b^{2}}{D}
\end{aligned}
$$
form as

$$
\begin{gathered}
y=0, u=1+\varepsilon e^{i t}, \theta=1+\varepsilon e^{i t}, C=1+\varepsilon e^{i t} \\
y=1, u=0, \theta=0, C=0
\end{gathered}
$$

The system of equations (11) - (13) together with the boundary conditions (14) - (15) constitutes the non dimensional form of the present model.

\section{Analytical Solution of the Problem}

To find the analytical solution of the non dimensional form of the present model we consider equations (11) - (15) and we assume the amplitudes of the free stream velocity, temperature and concentration variation are very small quantities. Hence, the amplitude of free stream velocity needs to be considered $0<\varepsilon \ll 1$

Using perturbation techniques the solution of the model has the following form

$$
\begin{aligned}
& u(y, t)=u_{0}(y)+\varepsilon u_{1}(y) e^{i t} \\
& \theta(y, t)=\theta_{0}(y)+\varepsilon \theta_{1}(y) e^{i t} \\
& C(y, t)=C_{0}(y)+\varepsilon C_{1}(y) e^{i t}
\end{aligned}
$$

Also, the free stream velocity takes the form as

$$
U=1+\varepsilon e^{i t}
$$

On substituting equations (16) - (19) into (11) - (13), and equating harmonic and non-harmonic terms and neglecting higher orders of $\varepsilon$ we obtain

$$
\begin{gathered}
u_{0}{ }^{\prime \prime \prime}-\phi u_{0}{ }^{\prime \prime}+\psi=\left[\operatorname{Gr} \theta_{0}+G c C_{0}-M^{2}\left(u_{0}-1\right)\right] \chi \\
u_{1}^{\prime \prime \prime}-\Omega u_{1}^{\prime \prime}+\Gamma=\left[G r \theta_{1}+G c C_{1}-\left(M^{2}+i \omega\right)\left(u_{1}-1\right)\right] \mathrm{K} \\
\theta_{0}{ }_{0}+\theta_{o}\left[\frac{Q_{o} h^{2} 3 P r}{\rho C_{p} v(3+4 R)}\right]=0
\end{gathered}
$$
(14) - (15) obtained after non dimensionalization as

$$
\begin{aligned}
& y=0, u_{0}=1, u_{1}=1, \theta_{0}=1, \theta_{1}=1, C_{0}=1, C_{1}=1 \\
& y=1, u_{0}=0, u_{1}=0, \theta_{0}=0, \theta_{1}=0, C_{0}=0, C_{1}=0
\end{aligned}
$$

Up on solving equations (22) - (25) together with the boundary conditions $(26)-(27)$, we obtain the required analytical solutions as

$$
\begin{aligned}
& \theta_{0}(y)=A_{1} e^{\xi y}+A_{2} e^{-\xi y} \\
& \theta_{1}(y)=C_{1} e^{\beta x}+C_{2} e^{-\beta x} \\
& C_{0}(y)=d_{1} e^{\lambda_{1} y}+d_{2} e^{-\lambda_{1} y} \\
& C_{1}(y)=f_{1} e^{\lambda_{2} y}+f_{2} e^{-\lambda_{2} y}
\end{aligned}
$$

Various symbols are expressed in the Appendix.

\section{Simulation Study of the Problem}

Here simulation study of different flow parameters is conducted using Mat lab code. We consider fluid flow bounded between two infinite vertical Couette channel walls embedded in a porous medium when the temperature and concentration oscillates with time in the presence of thermal radiation, chemical reaction, permeability of porous medium and electrically conducting viscoelastic fluid. For simplicity we have not presented the graphs of skin friction, Nusselt number, Prandtl number, mass diffusivity and Sherwood number.

The effects of physical parameters such as viscoelastic parameter, permeability of porous medium, thermal Grashof number, modified Grashof number, Hartmann number, frequency parameter, radiation parameter, chemical reaction parameter and Schmidt number on flow variables such as velocity, temperature and concentration of the model have been studied using simulation. 


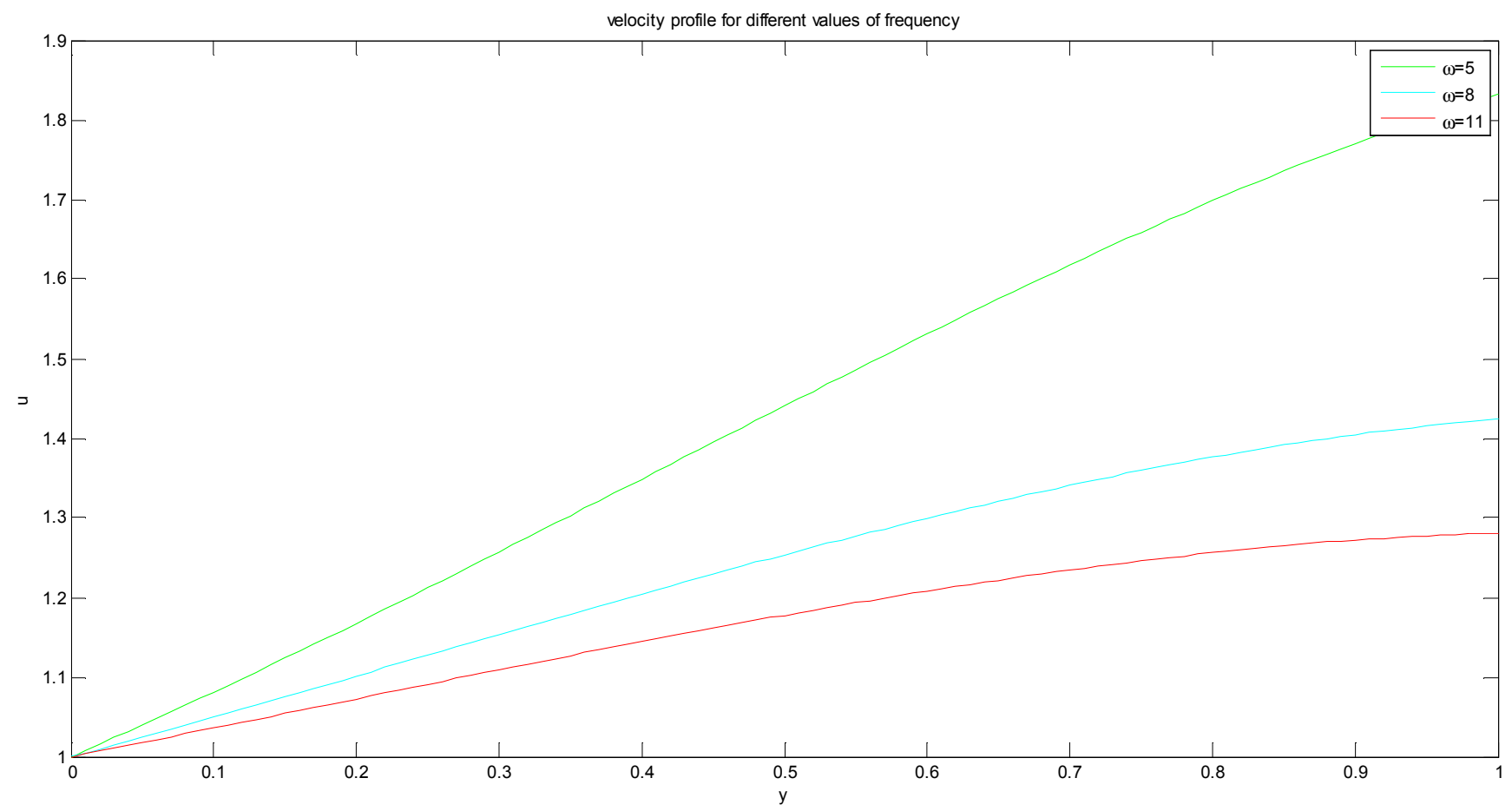

Figure 2. Velocity profile of the model for different values of frequency $(\omega)$.

In Figure 2, the simulated results of the influence of frequency parameter on velocity have been presented. The graph in $y u$ plane represents respectively the distance between the channel walls and velocity. Other parameters are held constant. It can be observed that the velocity $u$ starts with a minimum value near the moving channel wall and then it increases as it goes towards the stationary channel wall. Furthermore, the velocity decreases with increasing values of frequency.

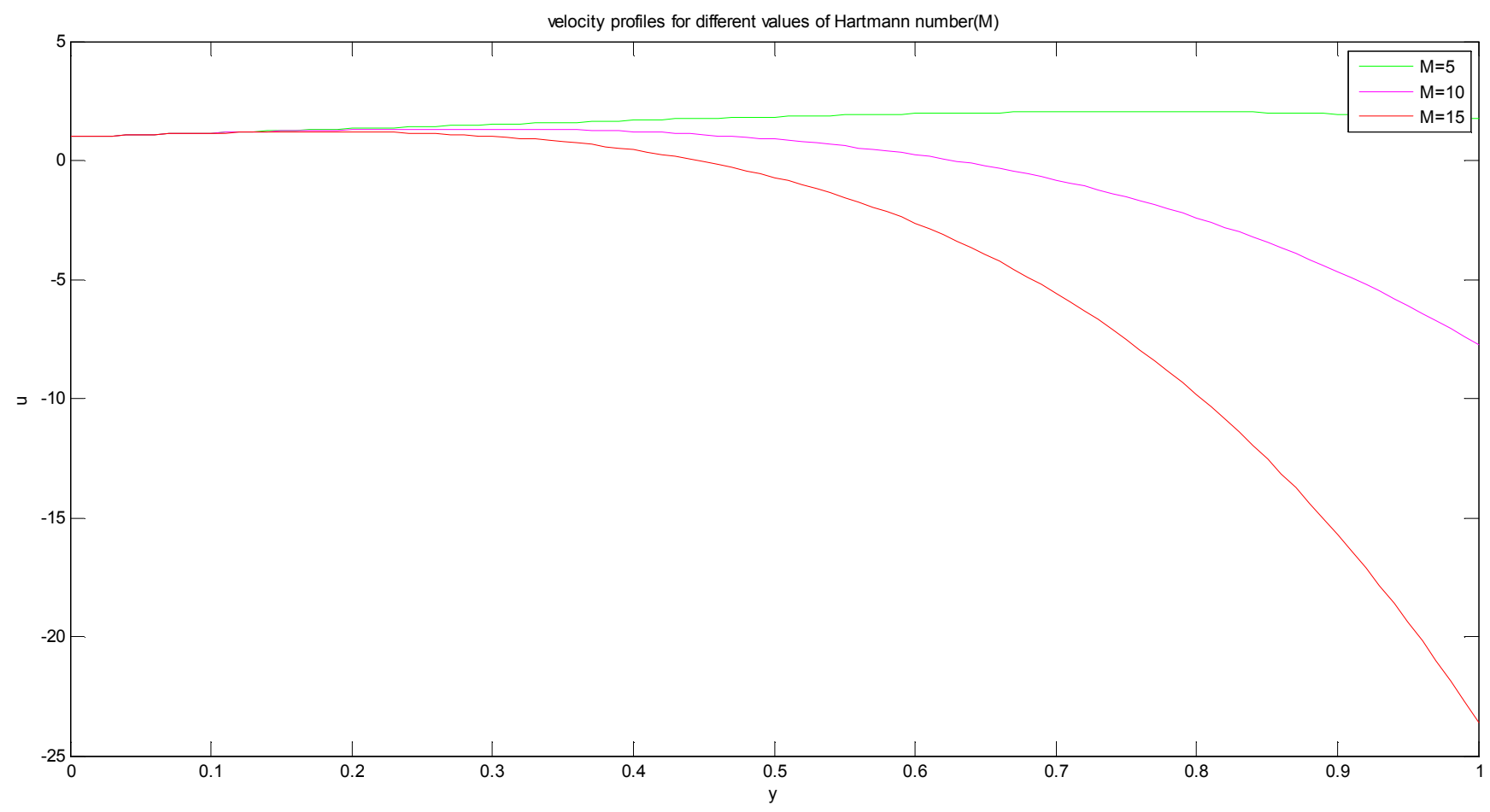

Figure 3. Velocity profile of the model for different values of Hartmann number $(M)$.

In Figure 3, the simulated results of the influence of Hartmann number on velocity have been presented. The graph in $y u$ plane represents respectively the distance between the channel walls and velocity. Other parameters are held constant. From the figure it is observed that if the value of Hartmann number $M$ is increased more than the strength of 
resistance of the magnetic field of the fluid flow will also increase more; and as a result the velocity also will decrease more. Furthermore, the decrement in velocity results in decreasing the temperature. It can be put in simple words that whenever the Hartmann number increases then the velocity as well as thermal boundary layer will decrease.

In Figure 4, the simulated results of the influence of viscoelastic parameter $k_{0}$ on velocity have been presented. The graph in $y u$ plane represents respectively the distance between the channel walls and velocity. Other parameters are held constant.

The result shows that for a fixed value of viscoelastic parameter $k_{0}$, the value of the fluid velocity denoted by $u$ starts from a minimum constant value at the moving plate, and as $y$ increases the velocity $u$ increases and reaches a maximum constant value at the stationary channel wall. Generally, it can be concluded from the simulation study that as viscoelastic parameter $k_{0}$ increases the velocity profile decreases i.e. flow of the fluid retards with increasing of viscoelastic parameter.

In Figure 5, the simulated results of the influence of temperature $\theta$ on velocity have been presented. The graph in $y u$ plane represents respectively the distance between the channel walls and velocity. Other parameters are held constant.

The result shows that for a fixed value of temperature $\theta$, the value of the fluid velocity denoted by $u$ starts from a minimum constant value at the moving plate, and as $y$ increases the velocity $u$ increases and reaches a maximum constant value at the stationary plate. Generally, it can be concluded from the simulation study that as temperature $\theta$ increases the velocity increases.

In Figure 6, the simulated results of the influence of thermal Grashof number $\mathrm{Gr}$ on velocity have been presented. The graph in $y u$ plane represents respectively the distance between the channel walls and velocity. Other parameters are held constant.

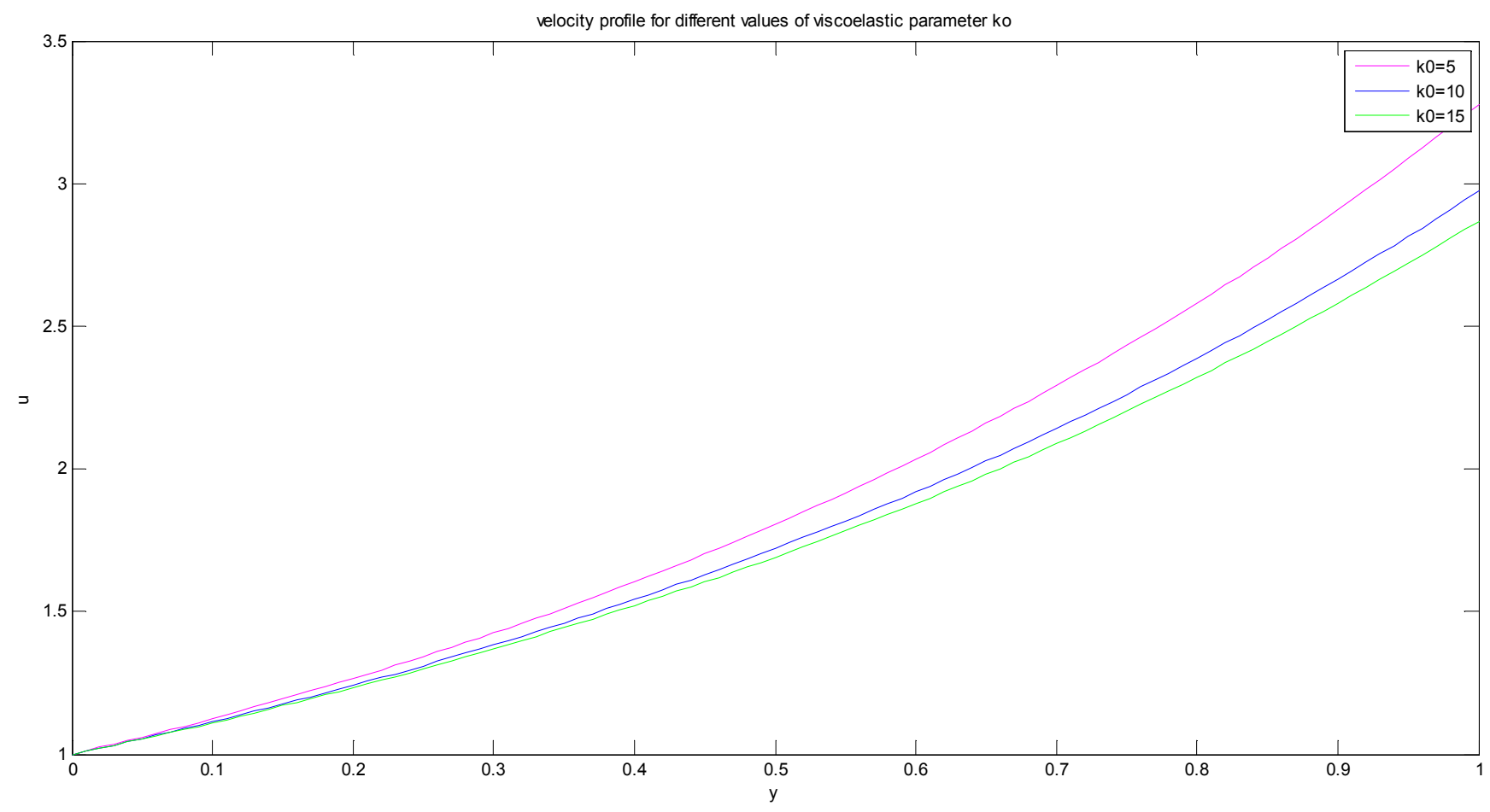

Figure 4. Velocity profile of the model for different values of viscoelastic parameter $\left(k_{o}\right)$.

The result shows that for a fixed value of thermal Grashof number $G r$, the value of the fluid velocity denoted by $u$ starts from a minimum constant value at the moving channel wall, and as $y$ increases the velocity $u$ increases and reaches a maximum constant value at the stationary channel wall. Generally, it can be concluded from the simulation study that as thermal Grashof number increases the velocity profile increases. Physically, this means that the increment of the buoyancy force leads to increase the vertical component of the velocity.

In Figure 7, the simulated results of the influence of Modified Grashof number $G c$ on velocity have been presented. The graph in $y u$ plane represents respectively the distance between the channel walls and velocity. Other parameters are held constant.

The result shows that for a fixed value of Modified Grashof number $G c$, the value of the fluid velocity denoted by $u$ starts from a minimum constant value at the moving channel wall, and as $y$ increases the velocity $u$ increases and reaches a maximum constant value at the stationary channel wall. Generally, it can be concluded from the simulation study that velocity of the fluid increases as modified Grashof number increases. 


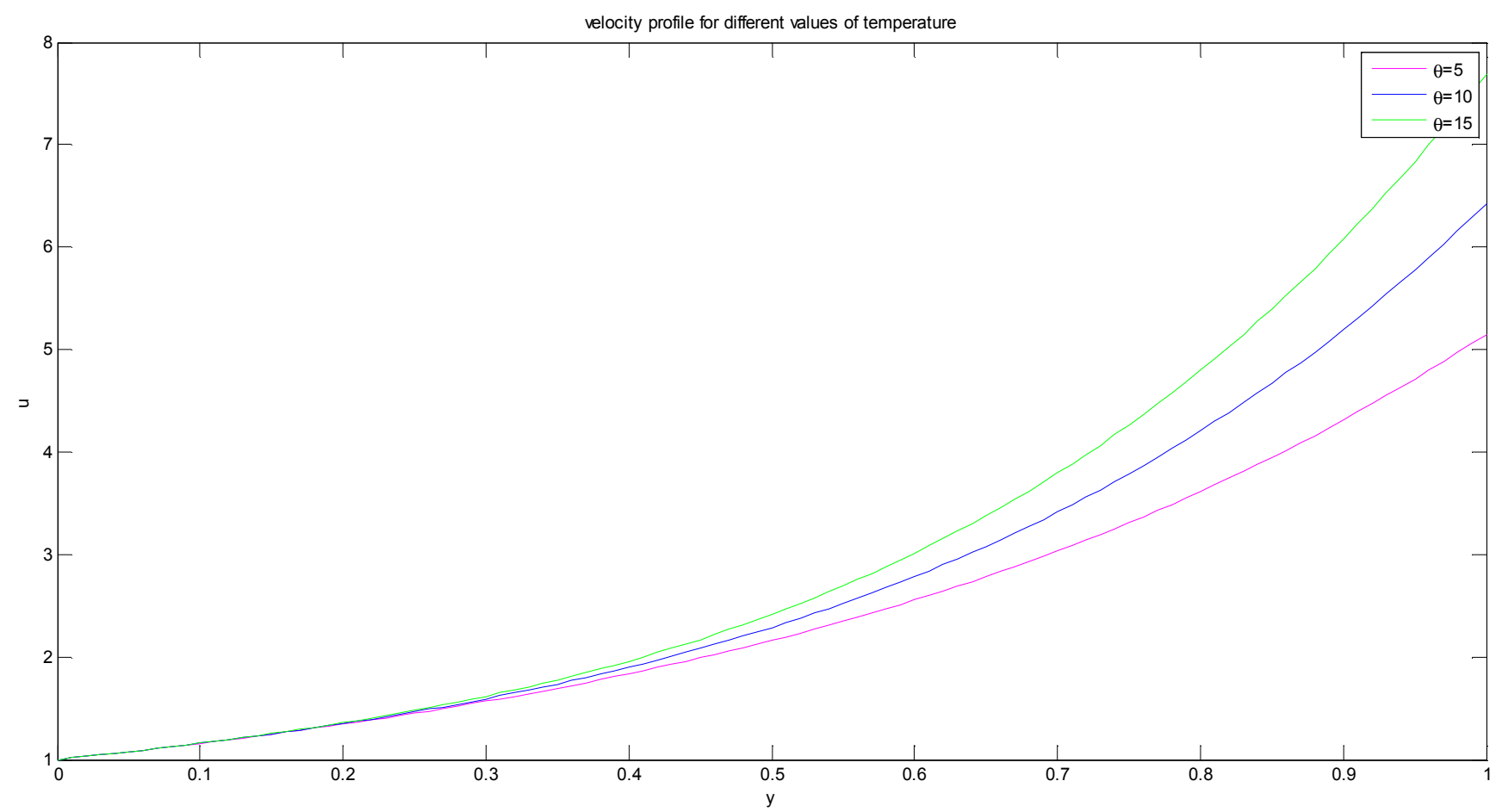

Figure 5. Velocity profile of the model for different values of temperature $(\theta)$.

In Figure 8, the simulated results of the influence of permeability of porous medium $k$ on velocity have been presented. The graph in $y u$ plane is representing respectively the distance between the channel walls and velocity. Other parameters are held constant.

The result shows that for a fixed value of permeability of porous medium $k$, the value of the fluid velocity denoted by $u$ starts from a minimum constant value at the moving channel wall, and as $y$ increases the velocity $u$ increases and reaches a maximum constant value at the stationary channel wall. Generally, it can be concluded from the simulation study that as permeability of porous medium increases the velocity of the fluid increases. Physically, this means that the resistance dominated by the porous medium reduces as the permeability of the medium increases because of which the velocity increases.

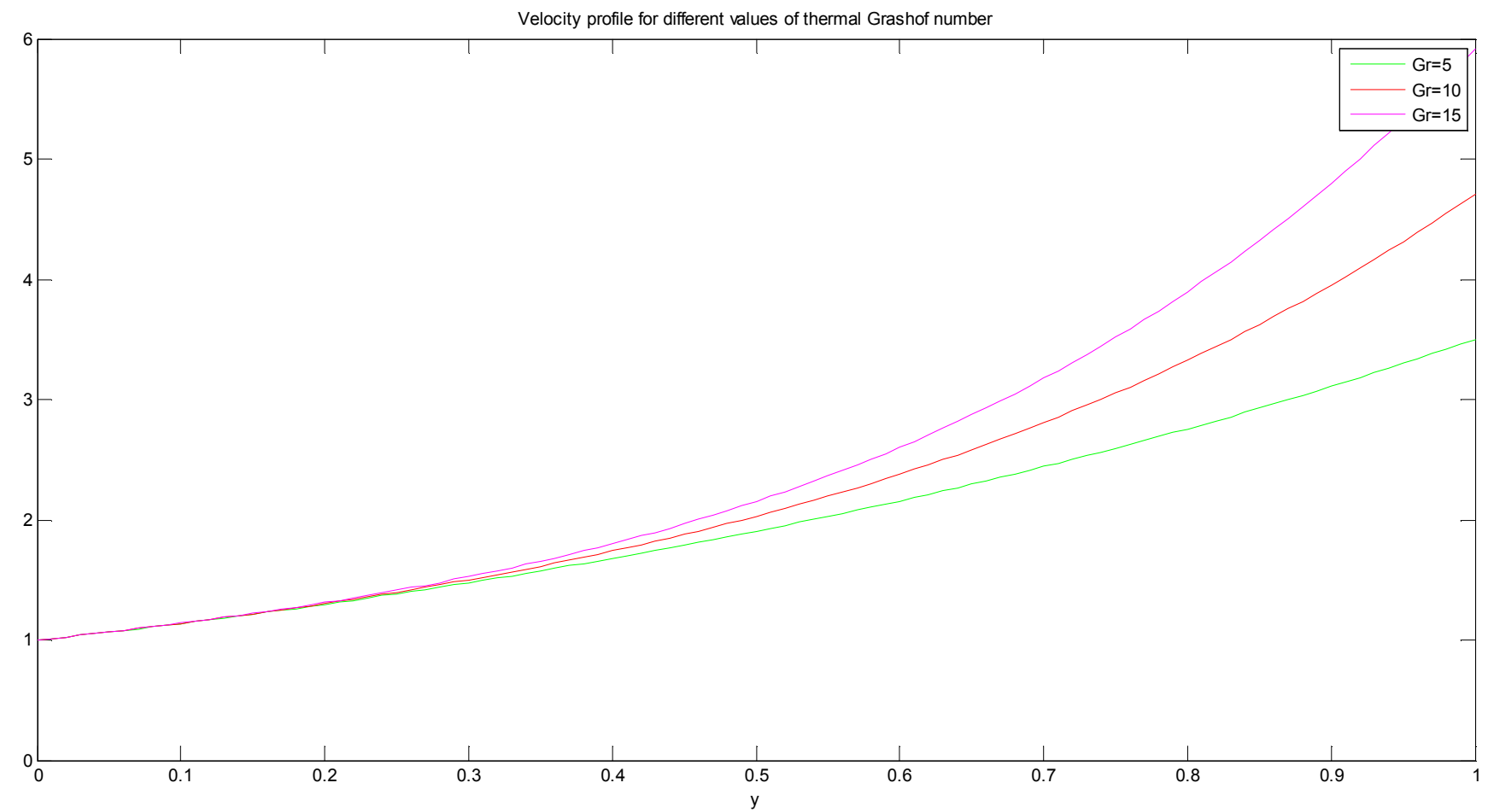

Figure 6. Velocity profile of the model for different values of thermal Grashof number (Gr). 
In Figure 9, the simulated result of the influence of Modified Grashof number $G c$ on the concentration of the fluid has been studied by simulation. Naturally, the number Gc and the concentration are inversely proportional to each other i.e. as $G c$ increases the velocity of the fluid increases and hence the concentration decreases. The same fact has been even supported by the present simulation study.

In Figure 10, the simulated result of the influence of radiation parameter $R$ on transfer of the temperature has been presented. The graph in $y \theta$ plane is representing respectively the distance between the channel walls and temperature. Other parameters are held constant.

The result shows that for a fixed value of thermal radiation parameter $R$, the temperature $\theta$ starts from a constant value at the moving channel wall. Also as $y$ increases the temperature decreases till it reaches a minimum value. Nevertheless, the measure of temperature at the moving channel wall is always at a higher value than that at the stationary channel wall.

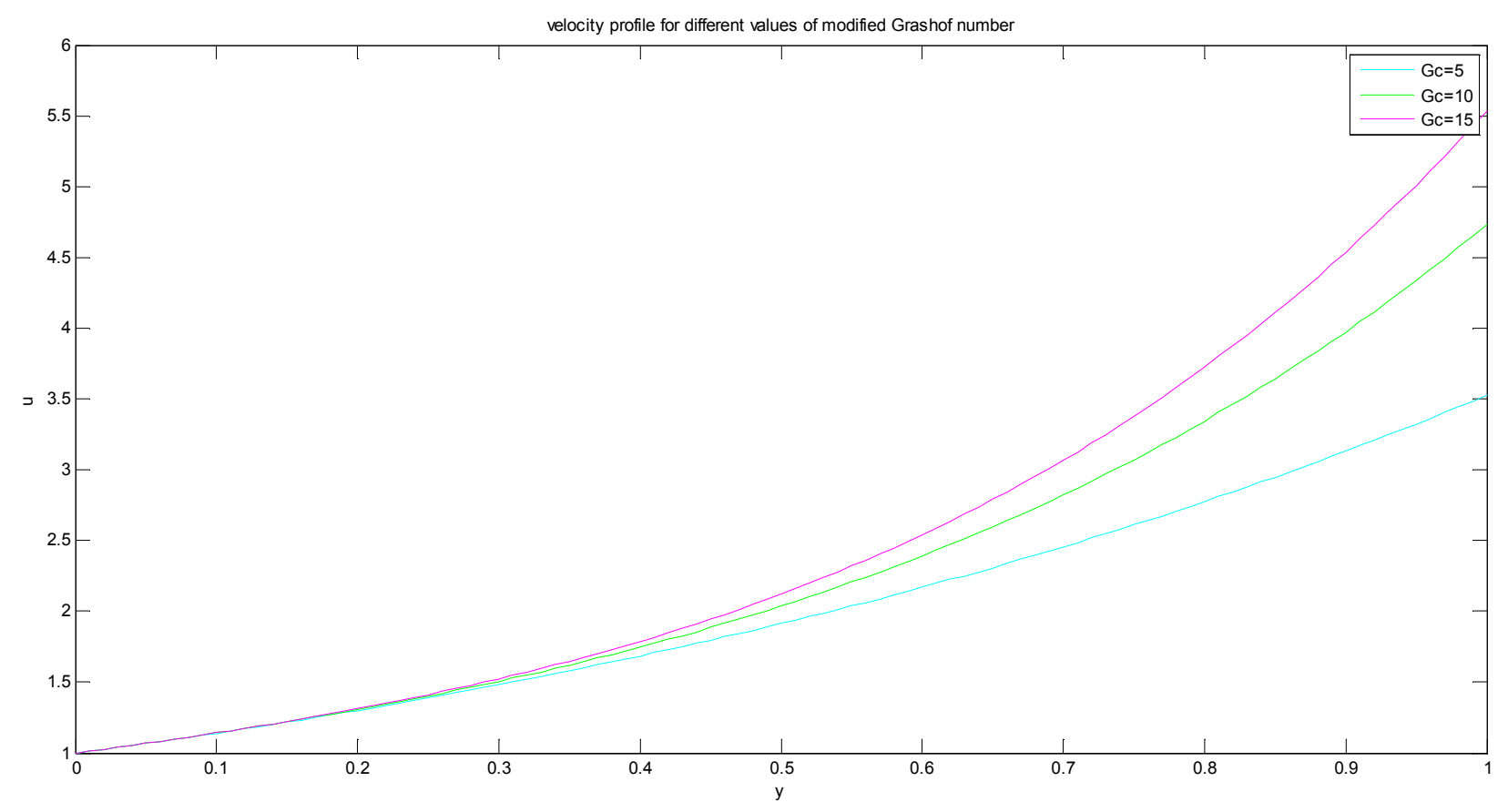

Figure 7. Velocity profile of the model for different values of Modified Grashof number (Gc).

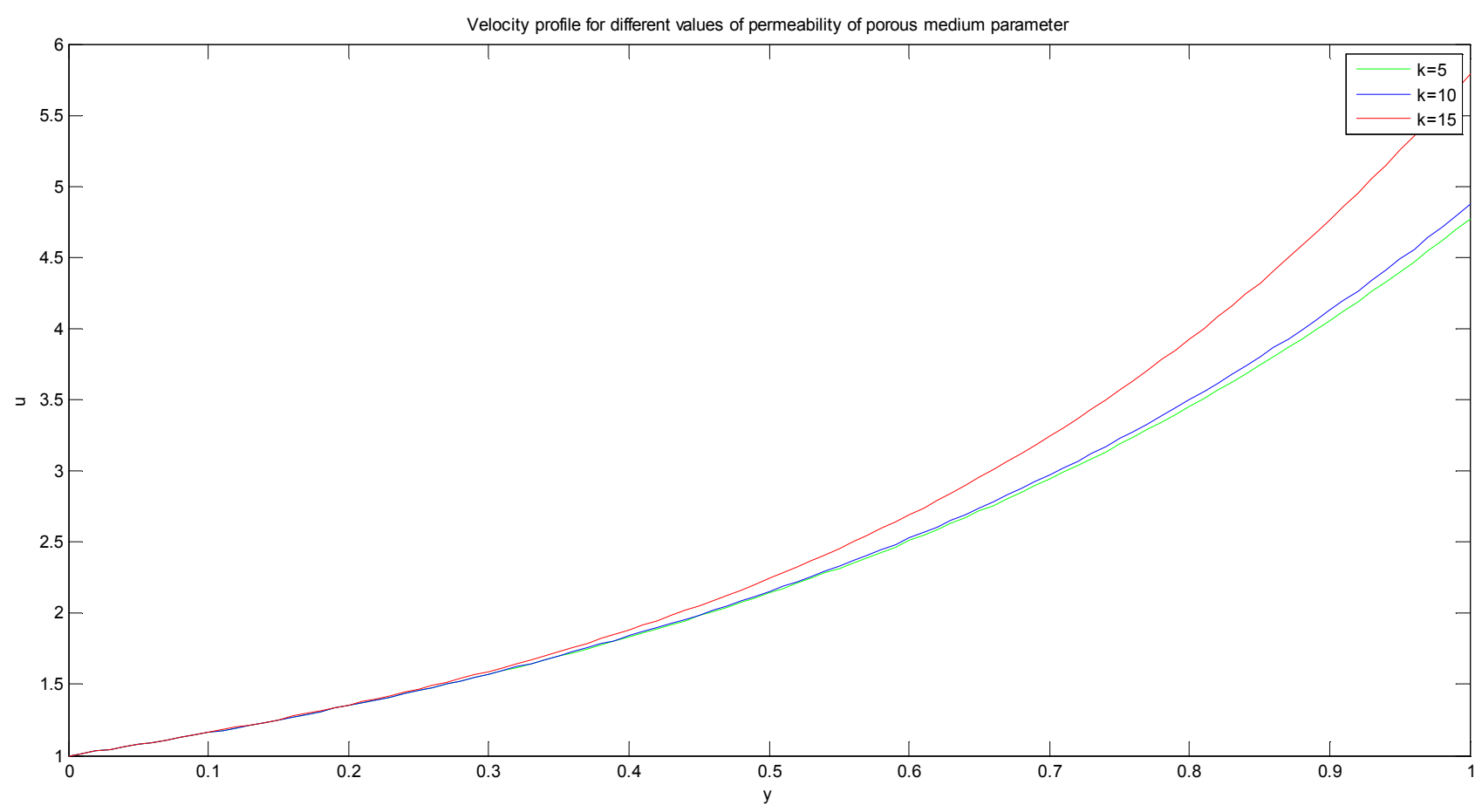

Figure 8. Velocity profile of the model for different values of permeability of porous medium parameter $(k)$. 
As the temperature of the moving channel wall is higher than that of the stationary channel wall, the temperature is expected to transfer from moving channel wall towards stationary channel wall. This fact has been supported in this simulation.

Also it can be observed from that simulation: (i) the value of temperature does not change with the change in $R$ at the moving channel wall, (ii) the minimum value of temperature, occurs between the two channel walls, decreases with the increase of $R$ i.e. minimum value of temperature is inversely proportional to that of $R$, and (iii) the value of temperature approaches zero with the increase of $R$ at the stationary channel wall.

In Figure 11, the simulated result of the influence of Chemical reaction parameter $K r$ on concentration has been presented. The graph in $y \beta$ plane is representing respectively the distance between the channel walls and concentration. Other parameters are held constant.

The result shows that for a fixed value of chemical reaction parameter $\mathrm{Kr}$, the value of fluid concentration denoted by $\beta$ starts from a maximum constant value at the moving, channel wall and as $y$ increases the concentration $\beta$ decreases and reaches zero constant value at the stationary channel wall. Nevertheless, the measure of fluid concentration at the moving channel wall is always at a higher value than that at the stationary channel wall.

As the concentration of fluid at the moving channel wall is higher than that at the stationary channel wall, the concentration is expected to move from moving channel wall towards stationary channel wall. Fluids diffuse from the locations of higher concentrations to that of lower concentrations. This fact has been supported in this simulation.

Also it can be observed from that simulation: (i) the value of concentration of fluid does not change with the change in $\mathrm{Kr}$ at the moving channel wall, (ii) the value of concentration of fluid, occurring between the two, channel walls decreases with the increase of $K r$ i.e. the value of concentration of fluid is inversely proportional to that of $K r$, and (iii) the value of concentration of fluid goes to zero at the stationary channel wall irrespective of the value of $K r$.

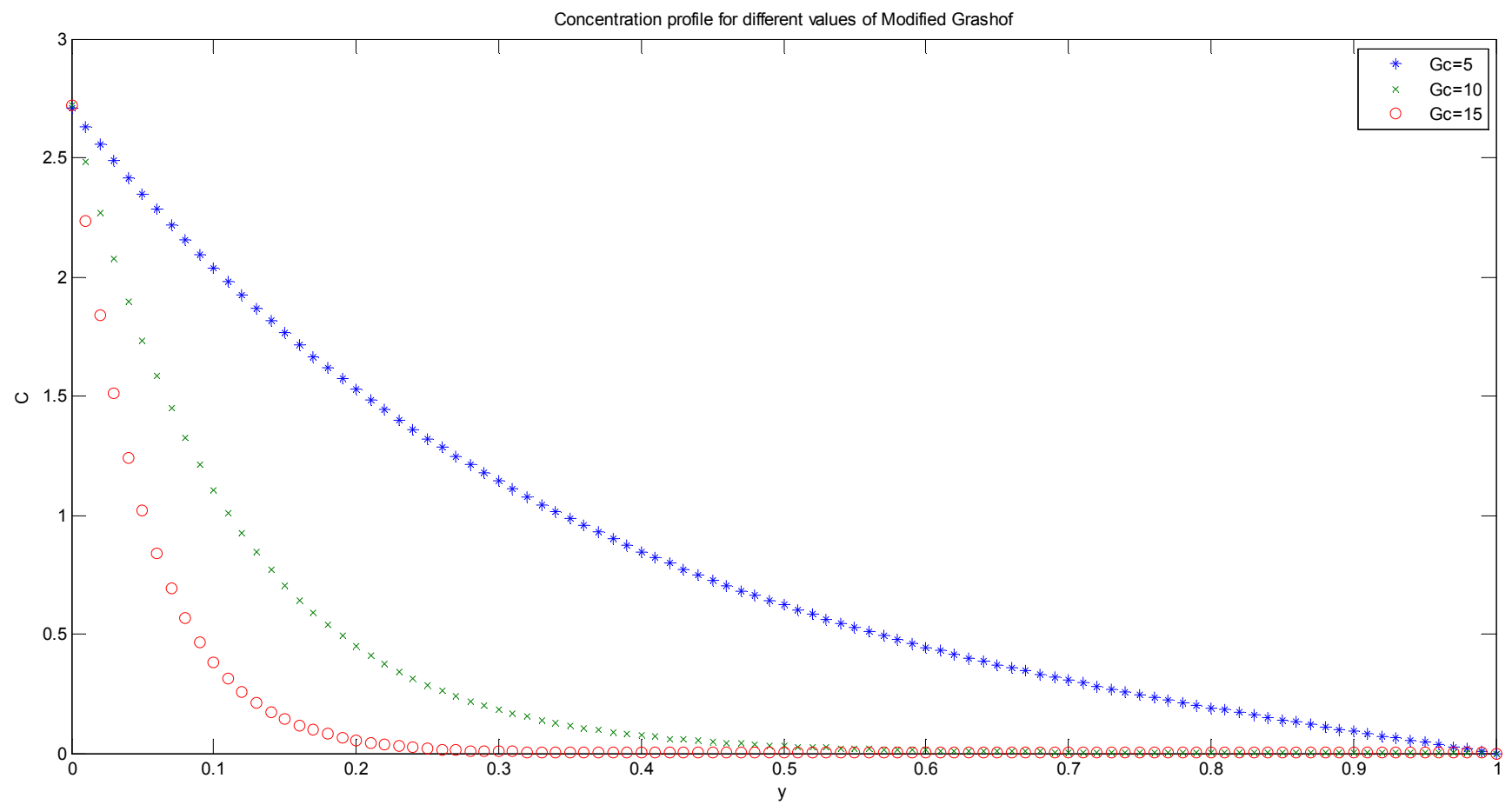

Figure 9. Concentration profile of the model for different values of Modified Grashof number (Gc).

In Figure 12, the simulated result of the influence of Schmidt number $S c$ on concentration has been presented. The graph in y co plane is representing respectively the distance between the channel walls and concentration. Other parameters are held constant.

The result shows that for a fixed value of Schmidt number $S c$ the value of fluid concentration denoted by $c o$ starts from a maximum constant value at the moving channel wall, and as $y$ increases the concentration co decreases and reaches zero constant value at the stationary channel wall. Nevertheless, the measure of fluid concentration at the moving channel wall is always at a higher value than that at the stationary channel wall.

As the concentration of fluid at the moving plate is higher than that at the stationary channel wall, the concentration is expected to move from moving channel wall towards stationary channel wall. Fluids diffuse from the locations of higher concentrations to that of lower concentrations. This fact has been supported in this simulation. 


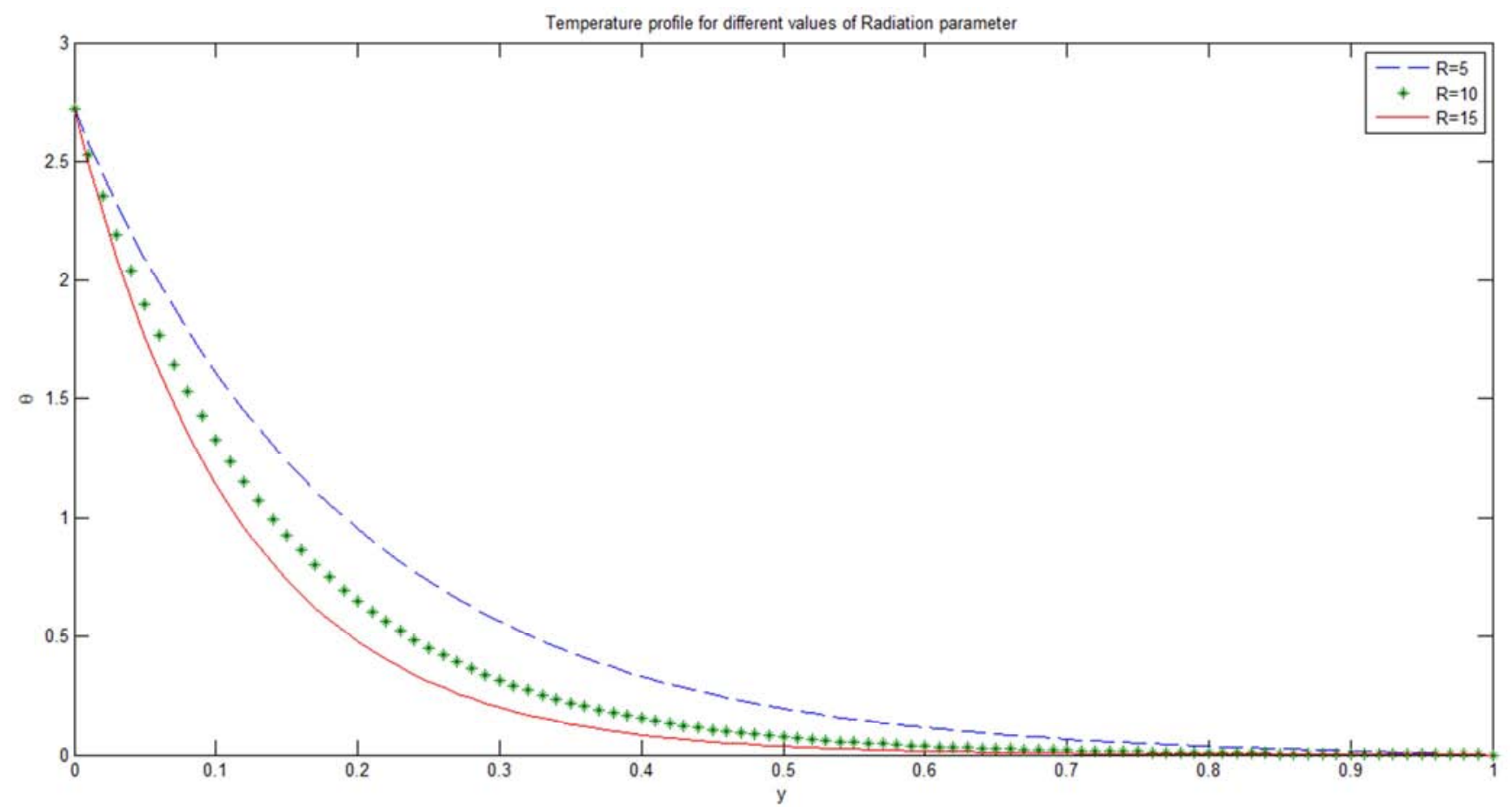

Figure 10. Temperature profile of the model for different values of Radiative parameter $(R)$.

Also it can be observed from that simulation: (i) the value of concentration of fluid does not change with the change in $S c$ at the moving channel wall, (ii) the value of concentration of fluid, occurring between the two channel walls, decreases with the increase of $S c$ i.e. the value of concentration of fluid is inversely proportional to that of $S c$, and (iii) the value of concentration of fluid goes to zero at the stationary channel wall irrespective of the value of $S c$.

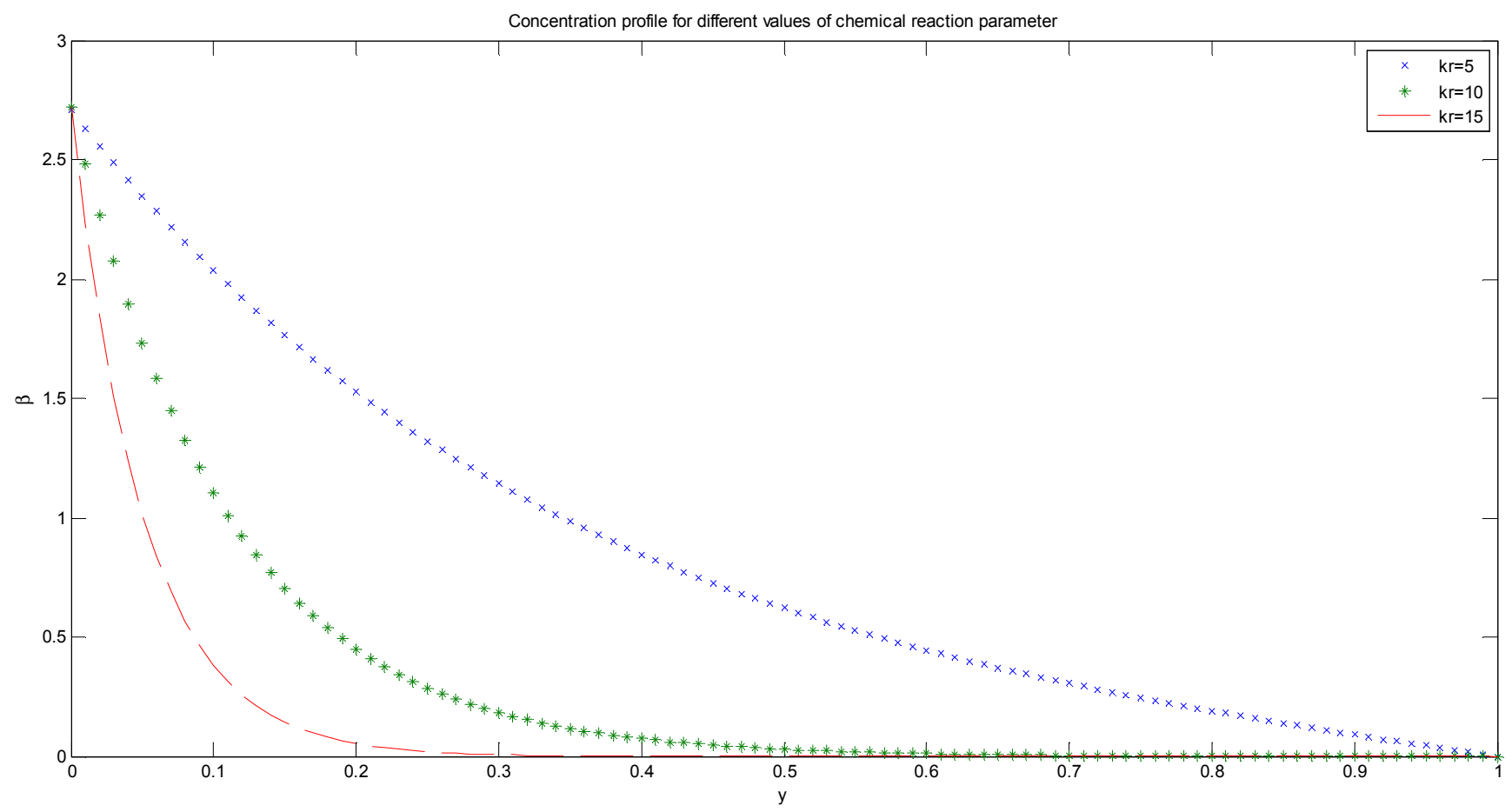

Figure 11. Concentration profile of the model for different values of chemical reaction parameter $(\mathrm{kr})$.

In general, the concentration decreases with an increase in $S c$. Physically, this is true since the increase in $S c$ implies decrease of molecular diffusivity implies decrease in concentration boundary layer. 


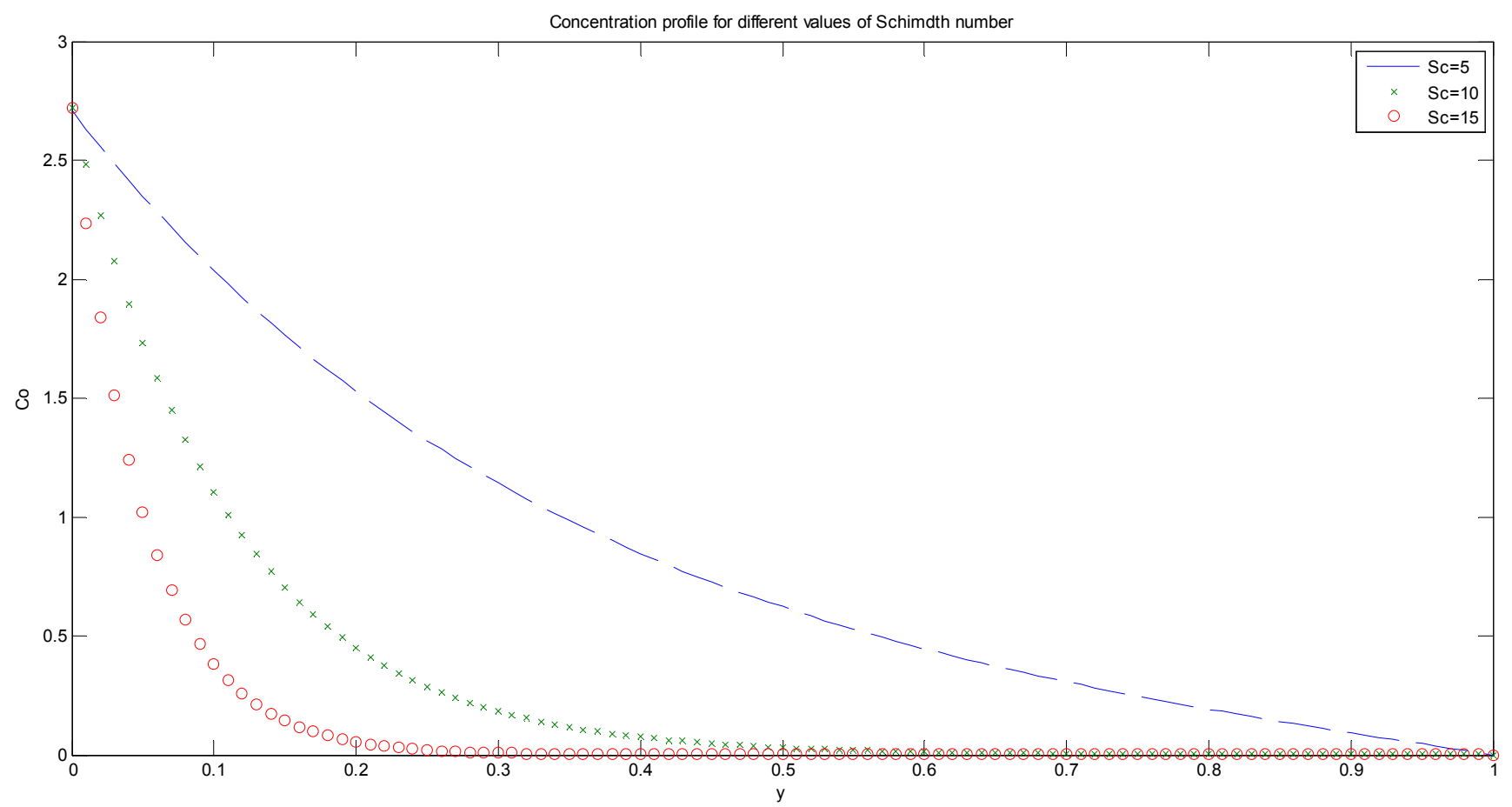

Figure 12. Concentration profile of the model for different values of Schmidt number (Sc).

\section{Conclusions}

In this paper the effect of physical parameters on flow variables of fluid flowing between a pair of infinite vertical Couette porous channel walls embedded in a porous medium is analyzed. The effects of physical parameters Viz. Hartmann number, Viscoelastic parameter, Permeability of porous medium, Chemical reaction parameter, radiative parameter, thermal Grashof number for heat transfer, modified Grashof number for mass transfer, frequency parameter and Schmidt number on flow variables Viz. velocity, temperature and concentration has been discussed. The solution of the governing equations is obtained using regular perturbation techniques. This technique is used to transform partial differential equations that are difficult to solve in closed form or explicit form. Mat lab code is used to solve and simulate the graphs of higher order ordinary differential equations. Hence we obtain the following results:

i. Velocity decreases with increasing values of frequency, Hartmann number, Viscoelastic parameter.

ii. Velocity increases with temperature, thermal Grashof number, modified Grashof number and permeability of porous medium.

iii. Thermal radiation parameter increases, the temperature decreases near the moving plate while it approaches to zero in the region near to the boundary layer of the stationary plate.

iv. An increment in both chemical reaction and Schmidt number results in decreasing in concentration.

\section{Appendix}

$$
\begin{aligned}
& \xi=\sqrt{\left(\frac{3 i P r}{4 R+3}\right) \frac{Q_{o} h^{2}}{\rho C_{p} v}}, C_{1}=\frac{1}{e^{2 \beta-1}}, \quad C_{2}=\frac{e^{2 \beta}}{e^{2 \beta-1}}, \lambda_{1}= \pm \sqrt{\frac{k_{r} b^{2}}{D}}, \\
& \beta=\sqrt{\left(\frac{3 i P r}{4 R+3}\right)\left(\frac{Q_{o} h^{2}}{\rho C_{p} v}-i \omega\right)}, \quad \lambda_{2}= \pm \sqrt{\frac{k_{r} b^{2}}{D}+i \omega S c} \\
& d_{1}=\frac{1}{1-e^{2 \lambda_{1}}}, \quad d_{2}=\frac{e^{2 \lambda_{1}}}{e^{2 \lambda_{1}}-1}, \quad f_{1}=\frac{1}{1-e^{2 \lambda_{2}}}, f_{2}=\frac{e^{2 \lambda_{2}}}{e^{2 \lambda_{2}-1}} \\
& \phi=\left(\frac{\alpha b^{2}}{v \mu \omega}\right)+\left(\frac{U_{0}^{2} \alpha b^{2}}{k_{0} v^{2} \mu^{2} \omega}\right), \quad A_{1}=\frac{-1}{e^{2 \xi}-1}, A_{2}=\frac{e^{2 \xi}}{e^{2 \xi}-1} \\
& \psi=\left(\frac{U_{0}^{4} u_{0} \alpha b^{4}}{\kappa v^{4} \mu^{2} \omega k_{0}}\right), \quad \chi=\left(\frac{U_{0}^{2} \alpha b^{2}}{k_{0} v^{2} \mu^{2} \omega}\right) \\
& \Omega=\left(\frac{\alpha b^{2}}{i v \mu \omega}\right)+\left(\frac{U_{0}^{2} \alpha b^{2}}{i k_{0} v^{2} \mu^{2} \omega}\right), K=\left(\frac{U_{0}^{2} \alpha b^{2}}{i k_{0} v^{2} \mu^{2} \omega}\right)
\end{aligned}
$$

\section{References}

[1] K. Walters, Non-Newtonian effects in some elastic-viscous liquids whose behavior at small rates of shear is characterized by a general linear equation of state. Quant. J. Mech. Appl. Math, 15, 1962, 63-76.

[2] Bhaskara Reddy N. and Bathaiah, D., Reg. J. of Energy Heat Mass Transfer, 3 (4), 1981, 239-255.

[3] Bhaskara Reddy N. and Bathaiah, D., Acta Mechanica, 42, 1982, 239-251.

[4] Chowdhury M. K. and Islam M. N., MHD-free convection flow of viscoelastic fluid past an infinite porous plate, Int. J. Heat Mass Trans., 36, 2000, 439-447. 
[5] Rajgopal K., Veena P. H. and Parvin V. K., Oscillatory Motion of an Electrically Conducting Viscoelastic Fluid over a Stretching Sheet in Saturated Porous Medium with Suction / Blowing. Mathematical Problems in Engineering, 1, 2006, 114.

[6] P. R. Sharma and D. Pareek, Unsteady flow and heat transfer through an elastic viscous liquid along an infinite hot vertical porous moving plate with variable free stream suction. Bull. Cal. Math. Sec. 98, 2006, 97-108.

[7] Pravat Kumar Rath, G. C. Dash, and P. K. Rath, Flow and heat transfer of an electrically conducting viscoelastic fluid between two horizontal squeezing/stretching plates. AMSE Modeling Measurement and Control 70, 2001, 45-63.

[8] S. M. B. Alhari, A. A. Mohamed and M. S. E. L. Gerdy, Heat and Mass transfer in MHD viscous elastic fluid flow through a porous medium over a stretching sheet with chemical reaction. Applied Mathematics 1, 2010, 446-455.

[9] B. Kumar and R. Sivaraj, MHD mixed convective viscoelastic fluid flow in a permeable vertical channel with Dufour effect and chemical reaction. Int. J. of Appl. Math. \& Mech. 14, 2011, 79-96.

[10] R. A. Damesh and B. A. Shannak, Viscoelastic fluid flow past infinite vertical porous plates in the presence of first order chemical reaction, Int. J. of Appl. Math. Mech. Engl. Ed. 31, 2010, 955-962.

[11] S. Dash, G. C. Dash and D. P. Mishra, MHD flow through a porous medium past a stretched vertical permeable surface in the presence of heat source/sink and a chemical reaction. Proc. Nat. Acad. Sci. India., 78A, 2008, 49-55.

[12] P. K. Rath, T. Parida and G. C. Dash, Three-Dimension free convective flow through porous medium in a vertical channel with heat source and chemical reaction. Proc. Nat. Acad. Sci. India, 82A, 2012, 225-232.

[13] P. R. Sharma and S. Sharma, Unsteady two dimensional flow and heat transfer through an elastic viscous liquid along an infinite hot vertical porous surface bounded by porous medium. Bull. Cal. Math. Sec. 97, 2005; 477-488.

[14] Arpita Mohanty, Pravat Kumar Rath, G. C. Dash, Unsteady MHD flow of a viscoelastic fluid a long a vertical porous surface with fluctuating temperature and concentration IOSR Journal of Engineering, ISSN (e); 2250-3021, ISSN (p); 22788719, 4, 2014, 46-57.

[15] Pawan Kumar Sharma, Mukesh Dutt, MHD oscillatory free convection flow past parallel plates with periodic temperature and concentration, Universal Journal of Applied Mathematics, 2 (7), 2014, 264 - 75, Doi: 10.13189/ujam.2014,020702.

[16] T. Sarpkaya, Flow of non-Newtonian fluids in a magnetic field, AICHE Journal 7, 1961, 324-328.

[17] Cogley A. C., Vinceti W. C. and Gilles S. E. Differential Approximation for Radiation Transfer in a Nongray Gas near Equilibrium. American Institute of Aeronautics and Astronautics Journal, 6, 1968, 551-555.

[18] Mansour M. A. Radiative and Free Convection Effects on the Oscillatory Flow past a Vertical Plate. Astrophysics and Space Science, 166, 1990, 269-275.

[19] Hossain M. A. and Thakar, H. S. Radiation Effect on Mixed Convection along a Vertical Plate with Uniform Surface Temperature. Heat and Mass Transfer, 314, 1996, 243-248.

[20] Hossain M. A., Alim M. A. and Rees S. The Effect of Radiation on Free Convection from a Porous Vertical Plate. International Journal of Heat and Mass Transfer, 42, 1999, 181-191.

[21] Muthucumarswamy R. and Senthil G. K., Effect of Heat and Mass Transfer on Moving Vertical Plate in the Presence of Thermal Radiation. Journal of Theoretical and Applied Mechanics, 31, 2004, 35-46.

[22] Aydin A. and Kaya A. Radiation Effect on MHD Mixed Convection Flow about a Permeable Vertical Plate. Heat and Mass Transfer, 45, 2008, 239-246.

[23] Muthucumarswamy R. and Janakiraman B. Mass Transfer Effects on Isothermal Vertical Oscillating Plate in the Presence of Chemical Reaction. International Journal of Applied Mathematics and Mechanics, 4, 2008, 66-74.

[24] Sudheer Babu M. and Satya Narayan P. V. Effects of the Chemical Reaction and Radiation Absorption on Free Convection Flow through Porous Medium with Variable Suction in the Presence of Uniform Magnetic Field. Journal of Heat and Mass Transfer, 3, 2009, 219-234.

[25] Makinde D. and Chinyoka T. Numerical Study of Unsteady Hydromagnetic Generalized Couette Flow of a Reactive Third-Grade Fluid with Asymmetric Convective Cooling. Computers and Mathematics with Applications, 61, 2011, 1167-1179.

[26] Skelland A. H. P. Non-Newtonian Flow and Heat Transfer, John Wiley, Sons, New York, 1976.

[27] Brewster M. A Thermal Radiative Transfer and Properties.New York: John Wiley and Sons, 1992. 